\title{
Two-photon intravital imaging of leukocyte migration during inflammation in the respiratory system
}

\author{
Young Min Kim, Soi Jeong, Young Ho Choe, Young-Min Hyun \\ Department of Anatomy and Brain Korea 21 PLUS Project for Medical Science, Yonsei University College of Medicine, Seoul, Korea
}

Two-photon intravital imaging is a powerful method by which researchers are able to directly observe biological phenomena in live organisms. Researchers in various biomedical research fields have applied two-photon imaging to a variety of target organs by utilizing this technology's ability to penetrate to significant depths with minimal phototoxicity. The mouse respiratory system in inflammation models is a good example, as two-photon intravital imaging can provide insights as to how the immune system is activated in response to inflammation within the respiratory system. Inflammation models can be generated via influenza viral, bacterial, or lipopolysaccharide injection. To exteriorize the lungs or trachea, thoracotomy or tracheotomy is performed, respectively; the appropriate combination of inflammation induction and organ exposure is selected depending on the study purpose. On the other hand, visualizing the movement of leukocytes is also an important component; to this end, immune cell populations of interest are either labeled via the genetic attachment of fluorescent proteins or stained with antibodies or dyes. With the proper selection of methods at each step, twophoton intravital imaging can yield visual evidence regarding immune responses to inflammation.

Key Words: inflammation; intravital imaging; respiratory system; two-photon microscopy

\section{INTRODUCTION}

The idiom "seeing is believing" is key to almost every scientific field, especially in biomedical science; advancements must always be accompanied by objective evidence that is readily observable. To this end, scientists have utilized various experimental methods, each with its own unique properties and advantages. Among such tools, intravital imaging using the twophoton confocal microscope has played a crucial role in unraveling novel biomedical processes at the cellular or molecular levels, particularly in the field of immunology. Intravital imaging provides researchers with a chance to observe actual phenomena within the organs of live animals, which consequently can lead to new biological advancements. Overall, research utilizing intravital imaging epitomizes the application of the aforementioned idiom, "seeing is believing," in science. In this review, we briefly introduce how two-photon intravital imaging would be useful to investigate immune cell migration in the respiratory system, including the lungs and trachea, under inflammation from the viewpoint of visualizing immune response. We also provide the principles of two-photon microscopy and surgery to expose the lungs or trachea for intravital imaging and explain how leukocyte subtype migration pat-

\section{Review Article}

Received: May 13, 2019

Accepted: May 17, 2019

Corresponding author

Young-Min Hyun

Department of Anatomy and Brain Korea 21 PLUS Project for Medical Science, Yonsei University College of Medicine, 50-1 Yonsei-ro, Seodaemun-gu, Seoul 03722, Korea

Tel: +82-2-2228-1655

Fax: +82-2-365-0700

E-mail:ymhyun@yuhs.ac

Copyright () 2019 The Korean Society of Critical Care Medicine

This is an Open Access article distributed under the terms of Creative Attributions Non-Commercial License (http:// creativecommons.org/li-censes/by-nc/4.0/) which permits unrestricted noncommercial use, distribution, and reproduction in any medium, provided the original work is properly cited. 
terns are visualized and analyzed in response to infection or inflammatory stimulation.

\section{TWO-PHOTON INTRAVITAL IMAGING}

\section{Principle of Two-Photon Microscopy}

Confocal fluorescence microscopy has been widely used for observing three-dimensional (3D) high-resolution fluorescent images in cells or tissues $[1,2]$. As a method of visualizing fluorescent images in cells or tissues using a single photon, confocal microscopy can penetrate up to $100 \mu \mathrm{m}$ within the tissue. In contrast, the more recently developed two-photon confocal imaging method focuses two near-infrared photon beams with longer wavelengths of around 700 to $1,000 \mathrm{~nm}$, enabling the imaging of tissues of up to $1,000 \mu \mathrm{m}$ in depth [3].

Since two-photon confocal imaging uses photons of long wavelengths and thus low energy to obtain image data, it causes less phototoxicity than conventional methods; therefore, imaging of both deeper depths and longer periods is possible [4]. Compared to traditional histological methods, which involve the isolation of tissues or cells from an organism followed by chemical treatment and ultimately imaging in a set period of time, intravital imaging using two-photon microscopy enables the researcher to study not only the structure but also the function of the target cell or tissue of a live animal. Taking advantage of such properties, much spotlight has been given to long-time 3D imaging of organs or tissues in live animals, mainly mice. To visualize target cells or tissue, mice are genetically modified to express fluorescent proteins or stained in specific cells and molecules or fluorescent antibody is used to label the target molecule. Intravital imaging using two-photon microscopy on animal models is an extremely useful technique that allows for real-time observation of cell movement and structure within the tissue and is widely utilized in immunology. It also enables visualization of cell movement and structure on a single-cell level, thus making direct observation of immune cell intravascular migration, extravasation, and interstitial migration possible, along with intracellular molecules that are involved in cell movement during such processes.

Thus, unlike conventional single-photon imaging methods, intravital imaging using two-photon confocal microscopy reveals more accurate and detailed information on cell-level movements within organisms and provides knowledge on the physiological and pathological roles of immune cells in inflammations and other diseases. As such, this imaging tool is being widely utilized in basic research that pursues novel therapeutic solutions.

\section{KEY MESSAGES}

- Two-photon intravital imaging would be useful to investigate immune cell migration in the lungs and trachea under inflammation.

- This review provides information on the principles of two-photon microscopy and surgery to expose the lungs or trachea for intravital imaging and also explains how leukocyte subtype migration patterns are visualized and analyzed in response to infection or inflammatory stimulation

\section{Current Trends in Intravital Imaging in Immunological Research}

For more than a decade, pioneering research in fields such as immunology has utilized intravital imaging to yield significant results. Especially, due to the ability of two-photon microscopy to penetrate up to $1,000 \mu \mathrm{m}$ in depth, intravital imaging has been frequently used in virtually every organ in research on mouse models of disease-including the respiratory system, lymphatic system, cremaster muscle, liver, and linings of the stomach, pancreas, kidney, spleen, heart, brain, and dermal tissue [5-9]. Depending on the study purpose, consecutive intravital imaging within various time periods is possible, ranging from 20 minutes to several hours.

On the other hand, one organ of interest can also be repeatedly imaged in the span of a few days. Therefore, long-term intravital imaging enables the observation of pathological and physiological phenomena involved with specific organs or tissues. Despite such advantages, two-photon confocal microscopy requires expensive equipment. In addition, even after acquiring such machinery, the actual surgery that exposes the organ of interest, along with methods of fixing the anesthetized organism to minimize the effect of heart pulsation and respiration, is also a task for the researcher. As of now, animal fixation equipment for brain tissue intravital imaging can be purchased; intravital imaging of other organs and tissues is mainly carried out with self-devised surgical methods and fixation equipment.

\section{Respiratory System Inflammation Model in Live Mice}

To study the biological phenomena that accompany inflammation, researchers have devised several different animal models. In particular, three infection methods have been frequently utilized to induce inflammation in mice; these include viral and bacterial infection as well as lipopolysaccharide (LPS) inoculation. For influenza viral infection, anesthetized mice 
are intranasally inoculated with an influenza A virus suspension. Intravital imaging of the trachea or the lungs can be performed between 1 and 5 days after infection, depending on the prognosis and immune cell subsets subject to investigation [10].

Similar to influenza viral infection, bacterial infection of the airway through the respiratory tract can be fatal. There are two common nosocomial pathogens that cause respiratory infection: Pseudomonas aeruginosa and Staphylococcus aureus. P. aeruginosa is an encapsulated, rod-shaped, Gram-negative bacterium that possesses the ability to cause opportunistic lung infections in patients with impaired immunity. It is wellknown that these infections lead to high mortality and morbidity in cystic fibrosis patients [11]. Therefore, these bacterial infection models of the airway of live mice would be of help to investigate actual responses of immune cells through intravital imaging. In general, wild-type P. aeruginosa strains, PAO1, and human-derived PA14 are generally used in animal infection models. The PA14 strain has a wide spectrum of infections from humans to animals and is virulent, unlike PAO1 [12]. The other common source of respiratory infection, S. aureus, is a round-shaped, Gram-positive bacteria that has several virulence factors including toxins and enzymes. Lipoteichoic acid and staphylococcal peptidoglycan are major components of $S$. aureus and are recognized by immune cells in a TLR2-dependent manner [13]. P. aeruginosa and S. aureus infection are correlated with the innate immune system in murine models; pathogen recognition and bacterial clearance of innate immune cells may play an important role in controlling P. aeruginosa and S. aureus [14]. It is very useful to perform intravital lung imaging of live animals for the investigation of the direct interaction between pulmonary innate immune cells and $P$. aeruginosa or S. aureus. From two-photon intravital microscopy, real-time dynamics of innate immune responsive phenomena for host defense such as neutrophil extracellular traps can be measured, which provides insight regarding the response mechanisms to infections $[15,16]$.

Furthermore, LPS has also been frequently employed as a perturbation element to the immune system. LPS, a major component of the outer membrane of Gram-negative bacteria, generates a response that accompanies cytokine activation from the immune system, contributing to the induction of sepsis $[17,18]$. Thus, the LPS-mediated mouse inflammation model can be used to confirm the activation of circulating leukocytes, migration of immune cells to tissues, and stimulation of these cells for cytokine release [19].

In mice lung inflammatory models, the two main methods of LPS injection are intratracheal and intranasal injection, respectively. While both methods are utilized to deliver the vehicle into the lungs, there exist differences in the injection procedure. Intratracheal injection is an administration method that allows a small amount of the vehicle to be directly delivered to the lungs or even to a single lobe in the lungs via bronchial catheter insertion. This method is particularly effective because of direct administration to the lungs, avoiding deposition of the vehicle in the nasal cavity [20]. On the other hand, intranasal injection takes advantage of respiration, using mice inhalation for vehicle delivery. This method is performed by gradual injection of the vehicle into the nostril of the mouse using a micropipette after anesthesia.

\section{Intravital Imaging of the Trachea and Lungs of the Respi- ratory System}

It is a crucial step in intravital imaging to exteriorize the target organ from the live animal. Not only should the proper organ be selected but also surgical methods that cause minimal harm to the organ deserve much scrutiny and practice. In terms of visualizing the respiratory system of mice, researchers often target either the trachea or lungs for imaging, depending on the specific goals of each study $[10,21]$. For intravital imaging of the mouse trachea, the anesthetized mouse is subsequently placed on a custom-designed platform for imaging. The trachea is exteriorized and a small cut is made on the frontal wall to insert an 18-gauge blunt-end cannula (Figure 1A) [10]. Various mouse strains can be used for exclusive visualization of the motility of particular immune cells; on the other hand, immune cell-specific fluorescent antibodies could also be intravenously injected to monitor circulating immune cells. Such cell visualization and trafficking methods will be discussed in more detail later.

Lung exposure is performed via thoracotomy, in which the thoracic cavity is opened to image the lung tissue. It is important to note that, while the thoracic cavity is opened, the pressure in the lung changes, making spontaneous breathing of the mouse difficult. Thus, prior to thoracotomy, intubation using an intravenous catheter is required [21,22]. A continuous supply of oxygen and isoflurane flows through the inserted catheter for life support during imaging. After intubation, skin and fatty tissue are removed to expose the thorax, the ribs are opened with surgical forceps, and the imaging apparatus is inserted. Finally, light vacuum pressure is applied to the apparatus to adsorb the lungs to the imaging window, and intravital imaging is conducted (Figure 1B). 


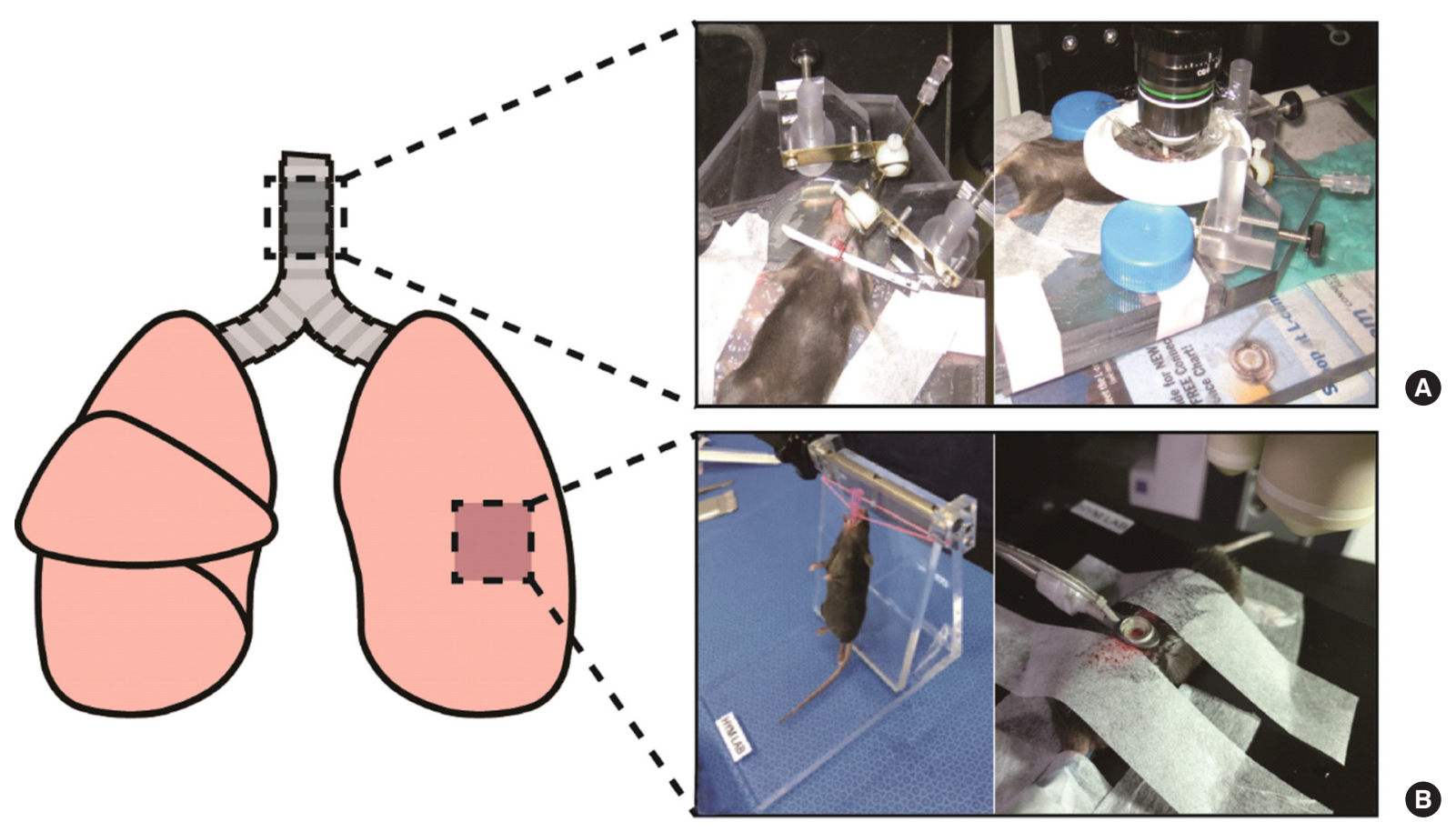

Figure 1. The site(s) of the lungs undergoing intravital imaging and the surgery pictures of each site. This figure schematically shows the respiratory organs of the mouse. (A) Images after trachea surgery show exposure of the trachea for intravital imaging. (B) Images show intubation step before lung surgery (left) and that the lung is absorbed in the imaging window after lung surgery (right).

\section{VISUALIZATION OF MIGRATION PATTERNS OF VARIOUS LEUKOCYTES}

\section{Tracking of Leukocyte Subtypes Using Fluorescent Protein Knock-in Mice}

To observe the movement and morphology of immune cells using intravital imaging, the labeling of immune cells is necessary. Numerous mouse strains have been generated and utilized for research, depending on the cell subset to be imaged. Lysozyme M-green fluorescent protein (LysM-GFP) mice have GFP attached to a particular type of lysozyme, which is an immune enzyme that degrades peptidoglycans in bacterial cell walls (Figure 2A). Lysozyme M, encoded by the Lyz2 gene, is expressed predominantly in neutrophils and also partly in macrophages; therefore, this strain is generally used for neutrophil visualization $[23,24]$. Another transgenic mouse line, the CX3Cr1-GFP mouse, is a strain with GFP attached to the CX3C chemokine receptor 1 (CX3Cr1), which is utilized as a marker for macrophages and monocytes $[25,26]$. Thus, CX3Crl-GFP mice are used for fluorescent visualization of monocytes and macrophages. While fluorescent labeling can be accomplished with the aforementioned methods, in some cases, it is prudent to visualize the flow of blood itself. Dextran is a commonly used blood vessel labeling material; for example, in mouse strains with GFP-labeled immune cell populations, Texas Red dextran could be used to stain blood vessels to create images with sufficient contrast between labeled immune cells and blood flow [27].

\section{Determination of Vascular Permeability Using Intravenously Injected Fluorescent Dextran}

To separately visualize blood vessels and leukocyte subtypes as described above, intravenous injection of fluorophore-conjugated dextran is widely used. Among various dextrans of different molecular weights, $70 \mathrm{kDa}$ dextran, which has a molecular weight similar to that of bovine serum albumin (66.5 $\mathrm{kDa})$, is particularly useful in visualizing blood vessels due to its tendency to flow along with blood circulation; in terms of color, injection of fluorophore (Texas Red [ $\mathrm{Ex}_{\max }, 596 ; \mathrm{Em}_{\max }=$ 615], fluorescein isothiocyanate [FITC; $\mathrm{Ex}_{\max }, 490$; $\left.\mathrm{Em}_{\max }, 525\right]$ ] conjugated dextran is specifically utilized for bloodstream visualization. While such tools offer a powerful method to image blood vessels and flow, vascular leakage from blood vessels to interstitial tissue could occur at stimulated or damaged vessels during the course of intravital imaging using fluorophoreconjugated dextran. Taking advantage of this phenomenon, the intensity of vascular leakage could also be quantified with the proper analysis software (e.g., Volocity, Imaris, ImageJ) [28]. 

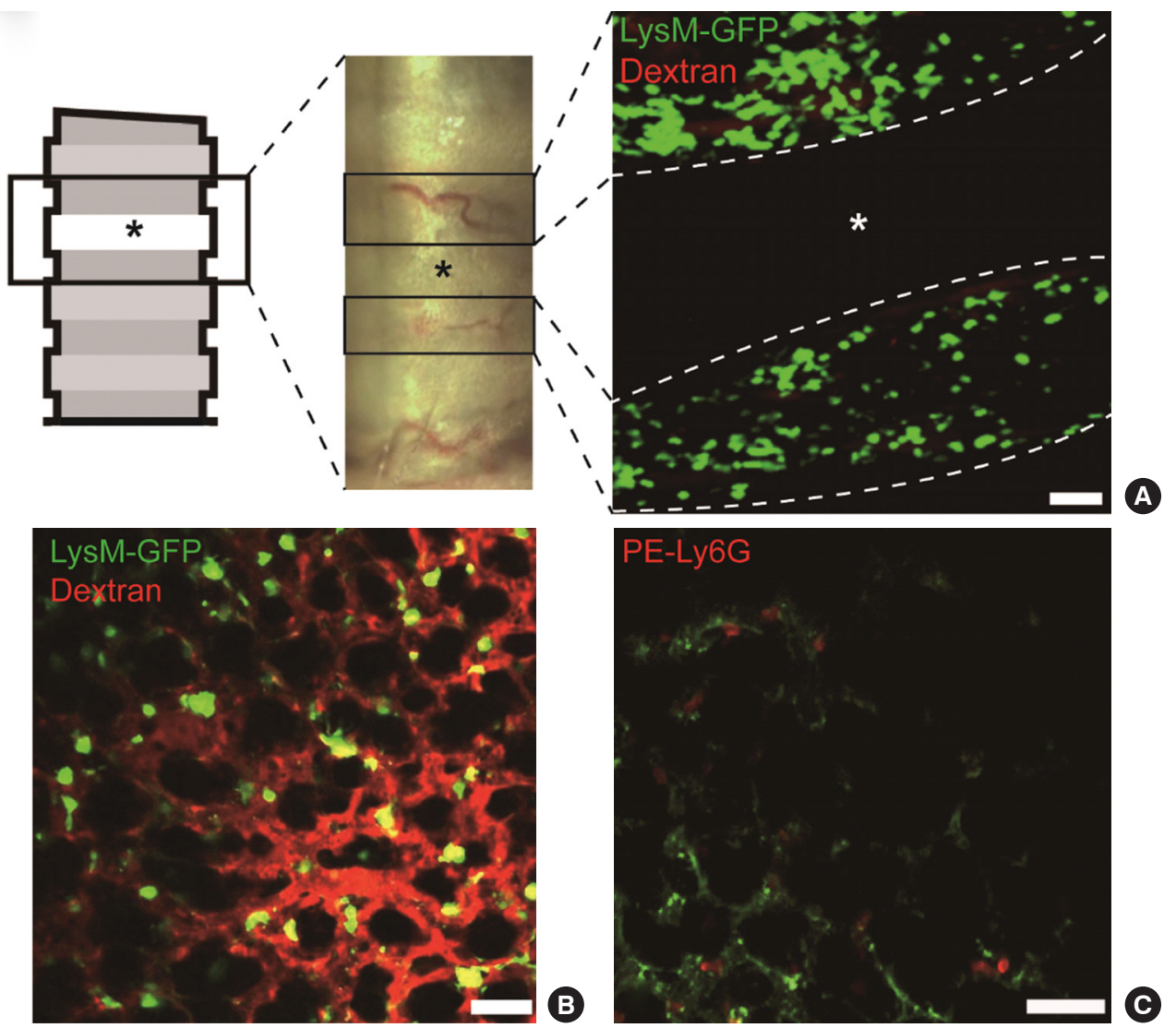

Figure 2. Tracheal and lung imaging obtained from intravital imaging. (A) The lung trachea is composed of annular ligament and cartilage. The annular ligaments (gray) and cartilage (white, asterisks) are seen in the box above the figure. There are no blood vessels in cartilage, so the images show neutrophils expressing lysozyme M-green fluorescent protein (LysM-GFP) and blood vessel-stained Texas Red dextran via intravital imaging at the annular ligament. Scale bar: $50 \mu \mathrm{m}$. (B) The image shows intravital imaging of a LysM-GFP mouse. The blood vessel is stained with Texas Red dextran. Scale bar: $50 \mu \mathrm{m}$. (C) The image shows intravital imaging using the neutrophil antibody PE-Ly6G (red) and the green structure reveals the autofluorescence of the lung. Scale bar: $50 \mu \mathrm{m}$.

\section{Tracking of Leukocyte Subtypes Using Fluorescent Antibody or Labeling Dye to Leukocyte Subtypes}

Another method of visualizing leukocyte subsets besides genetic strains is to use fluorescent antibodies or labeling dyes. Fluorescent antibody injection makes use of the property of antibodies to bind to specific antigens (Figure 2B, C). An example of a cell-surface protein that is abundantly expressed in murine neutrophils is Ly6G [29], while those expressed in macrophages or monocytes include Ly6C and F4/80 [30]. On antibodies that bind to these antigens, fluorescent dyes such as Alexa Fluor 488, FITC, or phycoerythrin are conjugated, allowing for separate visualization of cells according to wavelength [31].

\section{CONCLUSIONS}

As a powerful method to visualize cellular and molecular phenomena within live organisms, intravital imaging is being utilized in various fields of biology, across a wide variety of model animals and target organs. The mouse respiratory system is not an exception. Particularly, intravital imaging is extremely useful in observing leukocyte behavior in response to inflammation within mice. To this end, numerous methods to generate inflammation models have been devised, such as influenza viral, bacterial, and intratracheal or intranasal LPS injection. In addition, along with such inflammation model induction, the exposure of target organs is also crucial; two of the most frequently targeted organs are the trachea and lungs, which are exposed via tracheotomy and thoracotomy, respectively. With the proper inflammation model achieved and the appropriate surgery performed, the next step is to visualize the immune cell population of interest. Neutrophils, macrophages, and monocytes are generally labeled by two methods: knock-in mutations of fluorescent proteins such as the LysM- 
GFP or CX3Crl strains or staining using fluorescent antibodies and dyes. Such labeling methods could be improved at times with the simultaneous visualization of blood vessels using dextran; the proper combination of inflammation model, surgical method, and cell visualization will differ from study to study.

Intravital imaging is utilized in physiological and pathological research at a cellular and molecular levels in live animal models, and its usefulness is only expected to expand in the future. To that end, it is of paramount importance that researchers develop more efficient surgical methods and fixation equipment to inhibit the effects from heart pulses or respiration of the anesthetized animal. Furthermore, as of now, most researchers tend to use anesthetized organisms in intravital imaging, but a handful are reporting imaging results of brain tissues in unanesthetized mice [32,33]. This is probably an attempt to exclude effects of anesthesia, including changes in arterial pressure or blood flow, which may induce experimental errors. In the future, the development of more effective fixation methods is necessary for the intravital imaging of animals without anesthesia; however, since the organ to be imaged must still be exposed, a profound discussion regarding the pain of experimental subject animals is required. Accompanied with such theoretical and experimental advancements, intravital imaging will without doubt continue to contribute greatly in various research fields.

\section{CONFLICT OF INTEREST}

No potential conflict of interest relevant to this article was reported.

\section{ACKNOWLEDGMENTS}

This study was supported by a grant of the Ministry of Health \& Welfare, Republic of Korea (grant No. HI14C1324).

\section{ORCID}

Young Min Kim ～https://orcid.org/0000-0002-0452-2207

Soi Jeong https://orcid.org/0000-0002-2381-3325

Young Ho Choe https://orcid.org/0000-0001-7809-8165

Young-Min Hyun https://orcid.org/0000-0002-0567-2039

\section{AUTHOR CONTRIBUTIONS}

Conceptualization: YMH. Data curation: SJ, YMH. Formal analysis: SJ. Funding acquisition: YMH. Methodology: SJ, YHC. Project administration: YMH. Visualization: SJ, YHC. Writing - original draft: YMK, SJ, YHC, YMH. Writing - review \& editing: $\mathrm{YMH}$.

\section{REFERENCES}

1. Halin C, Mora JR, Sumen C, von Andrian UH. In vivo imaging of lymphocyte trafficking. Annu Rev Cell Dev Biol 2005;21: 581-603.

2. Beerling E, Ritsma L, Vrisekoop N, Derksen PW, van Rheenen J. Intravital microscopy: new insights into metastasis of tumors. J Cell Sci 2011;124(Pt 3):299-310.

3. Helmchen F, Denk W. Deep tissue two-photon microscopy. Nat Methods 2005;2:932-40.

4. König K. Multiphoton microscopy in life sciences. J Microsc 2000;200(Pt 2):83-104.

5. Honda T, Otsuka A, Kabashima K. Novel insights into cutaneous immune systems revealed by in vivo imaging. Allergol Int 2016;65:228-34

6. Fiole D, Tournier JN. Intravital microscopy of the lung: minimizing invasiveness. J Biophotonics 2016;9:868-78.

7. Basile DP, Friedrich JL, Spahic J, Knipe N, Mang H, Leonard EC, et al. Impaired endothelial proliferation and mesenchymal transition contribute to vascular rarefaction following acute kidney injury. Am J Physiol Renal Physiol 2011;300:F72133.

8. Coppieters K, Martinic MM, Kiosses WB, Amirian N, von Herrath $\mathrm{M}$. A novel technique for the in vivo imaging of autoimmune diabetes development in the pancreas by two-photon microscopy. PLoS One 2010;5:e15732.

9. Liang X, Grice JE, Zhu Y, Liu D, Sanchez WY, Li Z, et al. Intravital multiphoton imaging of the selective uptake of waterdispersible quantum dots into sinusoidal liver cells. Small 2015;11:1711-20.

10. Lim K, Hyun YM, Lambert-Emo K, Capece T, Bae S, Miller R, et al. Neutrophil trails guide influenza-specific $\mathrm{CD}^{+} \mathrm{T}$ cells in the airways. Science 2015;349:aaa4352.

11. Moradali MF, Ghods S, Rehm BH. Pseudomonas aeruginosa lifestyle: a paradigm for adaptation, survival, and persistence. Front Cell Infect Microbiol 2017;7:39.

12. Kukavica-Ibrulj I, Bragonzi A, Paroni M, Winstanley C, Sanschagrin F, O'Toole GA, et al. In vivo growth of Pseudomonas aeruginosa strains PAO1 and PA14 and the hypervirulent strain LESB58 in a rat model of chronic lung infection. J Bacteriol 2008;190:2804-13.

13. Fournier B, Philpott DJ. Recognition of Staphylococcus aure- 
us by the innate immune system. Clin Microbiol Rev 2005;18: 521-40.

14. Lavoie EG, Wangdi T, Kazmierczak BI. Innate immune responses to Pseudomonas aeruginosa infection. Microbes Infect 2011;13:1133-45.

15. Lefrançais E, Mallavia B, Zhuo H, Calfee CS, Looney MR. Maladaptive role of neutrophil extracellular traps in pathogen-induced lung injury. JCI Insight 2018;3.

16. Looney MR, Bhattacharya J. Live imaging of the lung. Annu Rev Physiol 2014;76:431-45.

17. Aderem A, Ulevitch RJ. Toll-like receptors in the induction of the innate immune response. Nature 2000;406:782-7.

18. Opal SM. Endotoxins and other sepsis triggers. Contrib Nephrol 2010;167:14-24.

19. Beutler B, Rietschel ET. Innate immune sensing and its roots: the story of endotoxin. Nat Rev Immunol 2003;3:169-76.

20. Osier M, Oberdörster G. Intratracheal inhalation vs intratracheal instillation: differences in particle effects. Fundam Appl Toxicol 1997;40:220-7.

21. Looney MR, Thornton EE, Sen D, Lamm WJ, Glenny RW, Krummel MF. Stabilized imaging of immune surveillance in the mouse lung. Nat Methods 2011;8:91-6.

22. Das S, MacDonald K, Chang HY, Mitzner W. A simple method of mouse lung intubation. J Vis Exp 2013;(73):e50318.

23. Faust N, Varas F, Kelly LM, Heck S, Graf T. Insertion of enhanced green fluorescent protein into the lysozyme gene creates mice with green fluorescent granulocytes and macrophages. Blood 2000;96:719-26.

24. Orthgiess J, Gericke M, Immig K, Schulz A, Hirrlinger J, Bechmann I, et al. Neurons exhibit Lyz2 promoter activity in vivo: implications for using LysM-Cre mice in myeloid cell research.
Eur J Immunol 2016;46:1529-32.

25. Garcia JA, Cardona SM, Cardona AE. Analyses of microglia effector function using CX3CR1-GFP knock-in mice. Methods Mol Biol 2013;1041:307-17.

26. Medina-Contreras O, Geem D, Laur O, Williams IR, Lira SA, Nusrat A, et al. CX3CR1 regulates intestinal macrophage homeostasis, bacterial translocation, and colitogenic Th17 responses in mice. J Clin Invest 2011;121:4787-95.

27. Noda S, Asano Y, Nishimura S, Taniguchi T, Fujiu K, Manabe I, et al. Simultaneous downregulation of KLF5 and Fli1 is a key feature underlying systemic sclerosis. Nat Commun 2014; 5:5797.

28. Park SA, Jeong S, Choe YH, Hyun YM. Sensing of vascular permeability in inflamed vessel of live animal. J Anal Methods Chem 2018;2018:5797152.

29. Lee PY, Wang JX, Parisini E, Dascher CC, Nigrovic PA. Ly6 family proteins in neutrophil biology. J Leukoc Biol 2013;94: 585-94.

30. Wynn TA, Chawla A, Pollard JW. Macrophage biology in development, homeostasis and disease. Nature 2013;496:44555.

31. Chiang EY, Hidalgo A, Chang J, Frenette PS. Imaging receptor microdomains on leukocyte subsets in live mice. Nat Methods 2007;4:219-22.

32. Gonçalves JT, Anstey JE, Golshani P, Portera-Cailliau C. Circuit level defects in the developing neocortex of Fragile X mice. Nat Neurosci 2013;16:903-9.

33. Issa JB, Haeffele BD, Agarwal A, Bergles DE, Young ED, Yue DT. Multiscale optical Ca2+ imaging of tonal organization in mouse auditory cortex. Neuron 2014;83:944-59. 